\title{
SEPARATION PROPERTIES FOR SELF-SIMILAR SETS
}

\author{
ANDREAS SCHIEF \\ (Communicated by Andrew Bruckner)
}

\begin{abstract}
Given a self-similar set $K$ in $\mathbb{R}^{s}$ we prove that the strong open set condition and the open set condition are both equivalent to $H^{\alpha}(K)>0$, where $\alpha$ is the similarity dimension of $K$ and $H^{\alpha}$ denotes the Hausdorff measure of this dimension. As an application we show for the case $\alpha=s$ that $K$ possesses inner points iff it is not a Lebesgue null set.
\end{abstract}

\section{INTRODUCTION}

In the study of fractal geometry, the first simple examples arise in the following manner: Given $N$ contracting similitudes $f_{i}, i=1, \ldots, N$, on $\mathbb{R}^{s}$ there exists a unique nonempty compact $K$ such that $K=\bigcup_{i=1}^{N} f_{i}(K)$, a so-called self-similar fractal. The first observation in this context is that the similarity dimension $\alpha$ and the Hausdorff dimension of $K$ (all terms are made precise in §1) coincide if the "pieces" $f_{i}(K)$ are pairwise disjoint. This result remains true if the pieces have only "small overlap", which is sometimes called "just touching". To describe this separation property Hutchinson gave the following definition: the open set condition (OSC) is fulfilled iff there is a nonempty open set $G$ such that the sets $f_{i}(G)$ are disjoint and contained in $G$. Since the intersection of $G$ and $K$ may be empty, Lalley strengthened the definition as follows: the strong open set condition (SOSC) holds iff furthermore $G \cap K \neq \varnothing$. Then the following chain of implications is well known $[3,4,6,7]$

$$
\mathrm{SOSC} \Rightarrow \mathrm{OSC} \Rightarrow H^{\alpha}(K)>0 \Rightarrow \operatorname{dim} K=\alpha
$$

(by $H^{\alpha}$ we mean the Hausdorff measure and by dim the Hausdorff dimension). An example due to Mattila clarifies that the last implication may not be inverted:

Take a nonconnected Sierpinski gasket in $\mathbb{R}^{2}$, i.e., for $i=1,2,3$ set $f_{i}(x)=$ $x_{i}+\left(x-x_{i}\right) / 3$ with $x_{1}=0, x_{2}=(1,0)$, and $x_{3}=(1 / 2,1 / 2 \sqrt{3})$. This fractal $K$ obviously fulfills the SOSC with $\alpha=1$. Therefore (see [3, 4]), Lebesgue almost all projections on a one-dimensional subspace of $\mathbb{R}^{2}$ (which are again self-similar sets) have dimension 1 but zero $H^{1}$ measure.

The question whether the other implications are equivalences was open. So several efforts were made to reduce in certain theorems the assumption SOSC

Received by the editors August 7, 1992 and, in revised form, November 18, 1992.

1991 Mathematics Subject Classification. Primary 28A78, 54E40; Secondary 54H15.

Key words and phrases. Self-similar sets, fractals, Hausdorff dimension. 
to OSC (see, e.g., [5]) or to give other characterizations for the positivity of $H^{\alpha}(K)$. Bandt and Graf developed in [1] an algebraic condition which is equivalent to $H^{\alpha}(K)>0$. Their paper was the starting point of this investigation, which will show that the first two implications are really equivalences.

Finally we will give an application of the main theorem to get in the case $\alpha=s$ the following proposition: $K$ has interior points iff $K$ is not a Lebesgue null set. The question whether in the "overlapping" case $\alpha>s$ each self-similar set with positive Lebesgue measure possesses interior points remains open.

\section{NotATION AND DEFINITIONS}

We consider $\mathbb{R}^{s}(s \geq 1)$ with the Euclidean metric $d$. The Hausdorff metric on closed subsets of $\mathbb{R}^{s}$ is denoted by the same letter. We set $D(F, K)=$ $\inf \{d(x, y): x \in F, y \in K\}$ for subsets $F$ and $K$ of $\mathbb{R}^{s} . A^{c}$ abbreviates the complement of $A$ and $\bar{A}$ the closure. $U(\varepsilon, x)$ is the open $\varepsilon$-neighborhood of $x$, and $U(\varepsilon, F)=\bigcup\{U(\varepsilon, x): x \in F\}$. The Lebesgue measure is denoted by $\lambda$ and the Hausdorff measure of dimension $\alpha$ by $H^{\alpha}$.

Given $N \in \mathbb{N}$ fix now similitudes $f_{1}, \ldots, f_{N}: \mathbb{R}^{s} \rightarrow \mathbb{R}^{s}$ with Lipschitz constants $\left.r_{i} \in\right] 0,1\left[\right.$, i.e., for $x, y \in \mathbb{R}^{s}, d\left(f_{i}(x), f_{i}(y)\right)=r_{i} d(x, y)$ holds. Let $K$ be the unique compact and nonempty set such that $K=\bigcup_{i=1}^{N} f_{i}(K)$.

We denote by $\mathscr{I}$ the set of all finite vectors $\mathbf{i}=\left(i_{1}, \ldots, i_{n}\right) \in\{1, \ldots, N\}^{n}$ with $n \geq 0$. The length of $\left(i_{1}, \ldots, i_{n}\right)$ is denoted by $|\mathbf{i}|=n$. Given $\mathbf{i}$ and $\mathbf{j}=\left(j_{1}, \ldots, j_{m}\right)$ we abbreviate $\mathbf{i j}=\left(i_{1}, \ldots, i_{n}, j_{1}, \ldots, j_{m}\right)$. $\mathbf{i}$ and $\mathbf{j}$ are incomparable if there exists no $\mathbf{k}$ such that $\mathbf{i}=\mathbf{j k}$ or $\mathbf{j}=\mathbf{i k}$.

The following abbreviations are important

$$
f_{\mathbf{i}}=f_{i_{1}} \circ \cdots \circ f_{i_{n}}, \quad K_{\mathbf{i}}=f_{\mathbf{i}}(K), \quad r_{\mathbf{i}}=r_{i_{1}} \cdots r_{i_{n}} .
$$

Furthermore, set $r_{\max }=\max \left\{r_{1}, \ldots, r_{n}\right\}$ and $r_{\min }=\min \left\{r_{1}, \ldots, r_{n}\right\} . \alpha$ is the unique solution of $\sum_{i=1}^{N} r_{i}^{\alpha}=1$. Finally we endow $\Omega=\{1, \ldots, N\}^{\mathbb{N}}$ with the product- $\sigma$-field and the canonical product measure

$$
\mathbb{P}=\bigotimes_{\mathbb{N}}\left(\sum_{i=1}^{N} r_{i}^{\alpha} \varepsilon_{i}\right),
$$

where $\varepsilon_{i}$ denotes the Dirac measure in point $i$. The image measure of $\mathbb{P}$ under the mapping

$$
\Omega \ni\left(i_{n}\right)_{n \in \mathbb{N}} \mapsto \lim _{n \rightarrow \infty} f_{\left(i_{1}, \ldots, i_{n}\right)}(0) \in K
$$

will be called $\mu$.

(1.1) Definition. We say that $\left(f_{1}, \ldots, f_{N}\right)$ (or, not completely correct, $K$ ) fulfills the open set condition (OSC) iff there exists an open nonempty set $G$ such that the sets $f_{i}(G), 1 \leq i \leq N$, are pairwise disjoint and all contained in $G$. If $G \cap K \neq \varnothing$ the strong OSC (SOSC) is fulfilled.

Note that without any further assumption $H^{\alpha}(K)<\infty$ holds for any selfsimilar set (cf. [6, p. 735]).

\section{THE THEOREM}

We start with the crucial implication. It has to be mentioned that the observation that $\gamma<\infty$ in the following proof is due to [1]. Nevertheless, we give the proof for the sake of completeness. 
(2.1) Theorem. If $H^{\alpha}(K)>0$ then the strong open set condition is fulfilled.

Proof. First of all, since the constants $r_{i}$ may be different, we have to give the following abbreviation: given $1 \geq b>0$ we set

$$
I_{b}=\left\{\mathbf{i}=\left(i_{1}, \ldots, i_{n}\right): r_{\mathbf{i}}<b \leq r_{\left(i_{1} \cdots i_{n-1}\right)}\right\} .
$$

The elements of $I_{b}$ are obviously incomparable and fulfill $K=\bigcup_{\mathbf{i} \in I_{b}} K_{\mathbf{i}}$, where the $K_{\mathbf{i}}$ are copies of $K$ of "approximately equal size".

(1) Let $x>0$. Then there exist open sets $U_{1}, \ldots, U_{n}$ such that

$$
U:=\bigcup_{i=1}^{n} U_{i} \supset K
$$

and

$$
\sum_{i=1}^{n}\left(\operatorname{diam} U_{i}\right)^{\alpha} \leq\left(1+x^{\alpha}\right) H^{\alpha}(K) .
$$

Let $\delta=D\left(K, U^{c}\right)$. We prove that for all incomparable $\mathbf{i}, \mathbf{j}$ such that $r_{\mathbf{j}}>x r_{\mathbf{i}}$ holds

$$
d\left(K_{\mathbf{i}}, K_{\mathbf{j}}\right) \geq \delta r_{\mathbf{i}} .
$$

Otherwise, since clearly $D\left(K_{\mathbf{i}}, f_{\mathbf{i}}(U)^{c}\right)=\delta r_{\mathbf{i}}$, we get $K_{\mathbf{j}} \subset f_{\mathbf{i}}(U)$. This implies

$$
\begin{aligned}
H^{\alpha}(K) r_{\mathbf{i}}^{\alpha}\left(1+x^{\alpha}\right) & <H^{\alpha}(K)\left(r_{\mathbf{i}}^{\alpha}+r_{\mathbf{j}}^{\alpha}\right)=H^{\alpha}\left(K_{\mathbf{i}}\right)+H^{\alpha}\left(K_{\mathbf{j}}\right) \\
& =H^{\alpha}\left(K_{\mathbf{i}} \cup K_{\mathbf{j}}\right) \leq \sum_{i=1}^{n}\left(\operatorname{diam} f_{\mathbf{i}}\left(U_{i}\right)\right)^{\alpha} \\
& =\sum_{i=1}^{n} r_{\mathbf{i}}^{\alpha}\left(\operatorname{diam} U_{i}\right)^{\alpha} \leq H^{\alpha}(K) r_{\mathbf{i}}^{\alpha}\left(1+x^{\alpha}\right),
\end{aligned}
$$

which is a contradiction. (The second to last inequality is a consequence of [1, Proposition 3], and the second equality may be seen as follows: Let $m$ be the maximal length of $\mathbf{i}$ and $\mathbf{j}$. Then the equality

$$
\sum_{|\mathbf{k}|=m} H^{\alpha}\left(K_{\mathbf{k}}\right)=\sum_{|\mathbf{k}|=m} H^{\alpha}(K) r_{\mathbf{k}}^{\alpha}=H^{\alpha}(K)
$$

implies that the intersection of two of these atoms is an $H^{\alpha}$-null set.)

(2) Let $1 / 3>\varepsilon>0$. We abbreviate (assuming without loss of generality $\operatorname{diam} K$ to be small enough to ensure $\left.\operatorname{diam} G_{k} \leq 1\right)$

$$
G_{\mathbf{k}}=U\left(\varepsilon r_{\mathbf{k}}, K_{\mathbf{k}}\right), \quad I(\mathbf{k})=\left\{\mathbf{i} \in I_{\operatorname{diam} G_{k}}: K_{\mathbf{i}} \cap G_{\mathbf{k}} \neq \varnothing\right\}, \quad \text { and } \quad \gamma=\sup _{\mathbf{k}} \# I_{\mathbf{k}}
$$

(where \# $C$ denotes the cardinality of $C$ ). We prove $\gamma<\infty$.

Since $r_{\mathbf{j}} \geq b r_{\min }>r_{\mathbf{i}} r_{\min }$ holds for $\mathbf{i}, \mathbf{j} \in I_{b}$, we may and do apply (1) for $x=r_{\min }$ to get $\delta>0$ such that $d\left(K_{\mathbf{i}}, K_{\mathbf{j}}\right) \geq \delta r_{\mathbf{i}}$ for arbitrary $b$ and different $\mathbf{i}, \mathbf{j} \in I_{b}$. This in turn implies the existence of $y \in K$ such that

$$
d\left(f_{\mathbf{i}}(y), f_{\mathbf{j}}(y)\right) \geq \delta r_{\mathbf{i}} .
$$

Given another point $z \in K$ such that $d(y, z)<\delta r_{\min } / 3$ we get $d\left(f_{\mathbf{i}}(y), f_{\mathbf{i}}(z)\right)$ $<r_{\mathrm{i}} \delta / 3$ and $d\left(f_{\mathrm{j}}(y), f_{\mathrm{j}}(z)\right)<r_{\mathrm{j}} \delta r_{\min } / 3<r_{\mathrm{i}} \delta / 3$, so $(*)$ holds also for $z$ with $\delta / 3$ instead of $\delta$. So we fix a finite set $Z \subset K$ whose $\delta / 3$ neighborhood 
covers $K$. Then for all $\mathbf{k}$ and all different $\mathbf{i}, \mathbf{j} \in I(\mathbf{k})$ there exists $z \in Z$ such that

$$
d\left(f_{\mathbf{i}}(z), f_{\mathbf{j}}(z)\right) \geq \delta r_{\mathbf{i}} / 3 \geq \delta d r_{\min } / 3
$$

(where $d:=\operatorname{diam} G_{k}$ ).

Intending to apply Ramsey's Theorem [2], we fix $z \in Z$ and consider $I \subset$ $I$ (k) such that for two members of $I$ the fixed $z$ fulfills the above inequality. All we have to do is to show that \# $I$ is bounded by a constant independent of $\mathbf{k}$. First we observe that the sets $U\left(\delta d r_{\min } / 6, f_{\mathbf{i}}(z)\right), \mathbf{i} \in I$, are disjoint and contained in $U\left(\operatorname{diam} K_{\mathbf{i}}+\delta d r_{\min } / 6, G_{\mathbf{k}}\right)$. This implies (using Lebesgue measure)

$$
\# I \leq \frac{\left(d+2 r_{\mathbf{i}} \operatorname{diam} K+\delta d r_{\min } / 3\right)^{s}}{\left(\delta d r_{\min } / 3\right)^{s}} \leq\left(\frac{1+2 \operatorname{diam} K+\delta r_{\min } / 3}{\delta r_{\min }}\right)^{s} .
$$

(3) Now choose $\mathbf{k}$ such that $\gamma=\# I(\mathbf{k})$. We show for arbitrary $\mathbf{j}$

$$
I(\mathbf{j k})=\{\mathbf{j i}: \mathbf{i} \in I(\mathbf{k})\} \text {. }
$$

By maximality, we only have to show "つ". But this is clear since $\varnothing \neq K_{\mathbf{i}} \cap G_{\mathbf{k}}$ implies

$$
\begin{aligned}
\varnothing & \neq f_{\mathbf{j}}\left(K_{\mathbf{i}} \cap G_{\mathbf{k}}\right)=f_{\mathbf{j}}\left(K_{\mathbf{i}}\right) \cap f_{\mathbf{j}}\left(G_{\mathbf{k}}\right)=K_{\mathbf{j i}} \cap f_{\mathbf{j}}\left(U\left(\varepsilon r_{\mathbf{k}}, K_{\mathbf{k}}\right)\right) \\
& =K_{\mathbf{j i}} \cap U\left(\varepsilon r_{\mathbf{j k}}, K_{\mathbf{j k}}\right)=K_{\mathbf{j i}} \cap G_{\mathbf{j k}} .
\end{aligned}
$$

Now let $l \neq j_{1}$. Since $K_{l}$ is covered by $\left\{K_{1}: 1 \in I_{\operatorname{diam} G_{\mathrm{jk}}}, l_{1}=l\right\}$ we immediately get

$$
D\left(K_{\mathbf{j k}}, K_{l}\right) \geq \varepsilon r_{\mathbf{j k}} .
$$

(4) Now we are in a position to define the desired separating open set

$$
U=\bigcup_{\mathbf{j} \in \mathscr{I}} G_{\mathbf{j k}}^{*}
$$

where $G_{\mathbf{i}}^{*}=U\left(\varepsilon r_{\mathbf{i}} / 2, K_{\mathbf{i}}\right)$. We get $K_{\mathbf{k}} \subset G_{\mathbf{k}}^{*} \subset U$, for each $i$ (cf. (3))

$$
f_{i}(U)=\bigcup_{\mathbf{j} \in \mathscr{I}} f_{i}\left(G_{\mathbf{j} \mathbf{k}}^{*}\right)=\bigcup_{\mathbf{j} \in \mathcal{I}} G_{i \mathbf{j} \mathbf{k}}^{*} \subset U,
$$

and finally for each $i \neq j$

$$
f_{i}(U) \cap f_{j}(U)=\varnothing .
$$

Indeed, if not, there are $\mathbf{i}$ and $\mathbf{j}$ such that $G_{i \mathrm{ik}}^{*} \cap G_{j \mathbf{k}}^{*} \neq \varnothing$ and $r_{i \mathrm{ik}} \geq r_{j \mathbf{j k}}$. If $y$ is an element of this intersection, there exist $y_{1} \in K_{i \mathbf{i k}}$ and $y_{2} \in K_{j \mathbf{j k}}$ such that $d\left(y, y_{1}\right)<\varepsilon r_{i \mathrm{ik}} / 2$ and $d\left(y, y_{2}\right)<\varepsilon r_{\mathbf{j k}} / 2$. We get $d\left(y_{1}, y_{2}\right)<\varepsilon r_{i \mathrm{ik}}$; hence, $D\left(K_{i \mathbf{i k}}, K_{j}\right)<\varepsilon r_{i \mathbf{i k}}$, which contradicts (3).

(2.2) Theorem. The following conditions are equivalent.

(i) SOSC holds with an open set $U$ such that $\mu(U)=1$.

(ii) SOSC.

(iii) $O S C$.

(iv) $H^{\alpha}(K)>0$.

(v) For each $\varepsilon>0, \gamma<\infty$ holds.

(vi) There exists $\varepsilon>0$ such that $\gamma<\infty$. 
Proof. The implications (i) $\Rightarrow$ (ii) $\Rightarrow$ (iii) and (v) $\Rightarrow$ (vi) are trivial; (iii) $\Rightarrow$ (iv) is standard; and (iv) $\Rightarrow(\mathrm{v})$ is included in the above proof. For the remaining implication (vi) $\Rightarrow(\mathbf{i})$ just set (continuing the proof above) $m=|\mathbf{k}|, U_{n}=$ $\bigcup_{|\mathbf{j}|=m n} G_{\mathbf{j k}}^{*}$, and $q=\mu\left(K_{\mathbf{k}}\right)$. The inequality

$\mu\left(\left(\bigcup_{n<l} U_{n}\right)^{c}\right) \leq \mathbb{P}\left(\left(i_{1}, \ldots, i_{m}\right) \neq \mathbf{k}, \ldots,\left(i_{(l-1) * m+1}, \ldots, i_{l m}\right) \neq \mathbf{k}\right)=(1-q)^{l}$ completes the proof.

(2.3) Corollary. Let $\alpha=s$. Then $K$ contains interior points if and only if $\lambda(K)>0$.

Proof. (1) Obviously only one implication requires a proof. Assume $\lambda(K)>0$, which means $H^{\alpha}(K)>0$, so by Theorem (2.1) we have an open set $U$ fulfilling the requirements of the OSC. Setting $G=U \cap U(1, K)$ we get a bounded open set with this property.

(2) We now prove $G \subset K$. An easy calculation yields

$$
\lambda\left(\bigcup_{i=1}^{N} f_{i}(G)\right)=\sum_{i=1}^{N} \lambda\left(f_{i}(G)\right)=\sum_{i=1}^{N} r_{i}^{s} \lambda(G)=\lambda(G)
$$

which implies at once that the open set $G \backslash \overline{\bigcup_{i=1}^{N} f_{i}(G)}$ is empty. Hence $\bar{G}=$ $\overline{\bigcup_{i=1}^{N} f_{i}(G)}$. Therefore,

$$
\bigcup_{i=1}^{N} f_{i}(\bar{G})=\bigcup_{i=1}^{N} \overline{f_{i}(G)}=\overline{\bigcup_{i=1}^{N} f_{i}(G)}=\bar{G}
$$

and, by the uniqueness of $K, \bar{G}=K$.

\section{ACKNOWLEDGMENT}

For a simplication in the proof of the corollary the author thanks S. Graf.

\section{REFERENCES}

1. C. Bandt and S. Graf, Self-similar sets 7. A characterization of self-similar fractals with positive Hausdorff measure, Proc. Amer. Math. Soc. 114 (1992), 995-1001.

2. C. Berge, Graphs and hypergraphs, North-Holland, Amsterdam, 1973.

3. K. J. Falconer, The geometry of fractal sets, Cambridge Univ. Press, New York, 1985.

4. __ Fractal geometry, Wiley, New York, 1990.

5. S. Graf, The equidistribution on self-similar sets, MIP-8929 Passau, 1989.

6. J. E. Hutchinson, Fractals and self-similarity, Indiana Univ. Math. J. 30 (1981), 713-747.

7. P. A. P. Moran, Additive functions of intervals and Hausdorff measure, Proc. Cambridge Philos. Soc. 42 (1946), 15-23.

Department of Mathematics, Ludwig-Maximilians-Universität, Theresienstrasse 39, 8000 Munich, GeRmany

Current address: Corporate Research and Development, SIEMENS AG, 81730 Munich, Germany

E-mail address: SCHIEF@CAYLEY.ZFE.SIEMENS.DE 